Article

\title{
Food Behaviors, Health, and Bean Nutrition Awareness among Low-Income Men: A Pilot Study
}

\author{
Michelle M. Heer and Donna M. Winham *(D) \\ Food Science \& Human Nutrition, Iowa State University, Ames, IA 50010, USA; mmheer@gmail.com \\ * Correspondence: dwinham@iastate.edu; Tel.: +1-515-294-5040
}

Received: 26 November 2019; Accepted: 5 February 2020; Published: 6 February 2020

\begin{abstract}
Bean consumption is known to lower blood cholesterol and postprandial blood glucose. With higher chronic disease risk, low-income men could theoretically benefit from increased bean intakes. The study objective was to explore low-income men's food behaviors, bean health benefit awareness, and bean consumption practices and preferences. Seventy-one low-income men aged 18-65 years ( $\mu 41 \pm 12.7 ; 53 \%$ white, $16 \%$ black, 31\% Hispanic) completed a survey on health risks, food behaviors, bean health knowledge, attitudes toward dry and canned beans, and bean preferences. Eighty-seven percent had one or more health risk factors of physical inactivity, smoking, or obesity. Most men compared food prices, and thought about healthy food choices for their families, but few planned meals or read nutrition facts labels. White men had significantly higher bean health benefit knowledge than black or Hispanic men $(p<0.01)$. Most men liked the taste of beans, disagreed dry beans took too long to prepare, and 79\% ate them at least 2-3 times per month. Forty-nine percent agreed beans caused intestinal gas. Improving men's awareness of the health benefits of beans as well as leveraging existing positive attitudes may be useful approaches to increase bean consumption among low-income and minority male populations.
\end{abstract}

Keywords: food behavior; nutrition knowledge; pulses; purchasing preferences; health disparities

\section{Introduction}

The leading causes of death among adult men in the United States (US) remain heart disease (24.4\%) and cancer $(21.9 \%$ ) [1]. The risk factors for these diseases as well as type 2 diabetes, hypertension, and obesity are mediated by lifestyle behaviors such as diet, physical activity, regular medical checkups, and not smoking [2]. Men may be less concerned about healthy dietary patterns and lack awareness of diet-health associations in comparison to women [3].

Along with gender, wage earnings and race/ethnicity have a considerable impact on health and disease outcomes [4]. Low-income men have increased prevalence of health risk factors including physical inactivity, smoking, and obesity, as well as increased chronic disease rates such as heart disease, stroke, diabetes, and cancer [4,5]. Disease rates are greater in blacks and Hispanics than non-Hispanic whites, yet within race/ethnicity groups, rates are linked inversely to income $[1,4,5]$.

Not unexpectedly, low-quality diets are associated with limited economic resources [6,7]. A lack of dietary diversity accompanied with high levels of saturated fat and low vegetable, fruit, and fiber intake contributes to disease risk [4]. Pulses, which include beans, peas, lentils and chickpeas, can improve dietary quality by providing protein, fiber, and many micronutrients associated with other vegetables, including folate, iron, zinc, magnesium, and potassium [8,9]. Compared to other foods, beans have one of the best nutrient-to-price ratios, making them ideal for maximizing nutrition with economic constraints [9]. Bean consumption is associated with both reduced risk and improved outcomes of cardiovascular disease [10], serum cholesterol [11], postprandial glucose control [12], type 
2 diabetes [13], and some cancers [14]. The dietary fiber in beans is likely a contributing component to lower risk of these chronic diseases [15].

The 2015-2020 Dietary Guidelines for Americans (DGA), which aim to promote health, prevent chronic disease, and help Americans maintain a healthy weight, suggests 2-3 cups of cooked beans per week for men ages 19-50 [2]. On average, men currently consume 1 cup per week [2]. Increasing bean consumption to meet the DGA could improve nutrient shortfalls such as fiber, potassium, and iron, and reduce disease-related risks [15,16]. Only a handful of published studies exist on the factors that influence bean consumption among Americans, and these are mostly with minorities or women [14,17-21]. Determining the views and current knowledge toward beans among low-income males is necessary to design tailored intervention programs to boost bean intake and improve health.

There were five study objectives. These were to determine low-income men's: (1) health risk factors; (2) food behavior practices; (3) knowledge of bean health benefits; (4) attitudes and perceptions toward dry and canned beans; and (5) consumption patterns and purchasing preferences of beans.

\section{Materials and Methods}

\subsection{Participants and Data Collection}

Low-income male clients, aged 18-65 years old, were recruited from an employment center, a Special Supplemental Nutrition Program for Women, Infants and Children (WIC) clinic, and classes of the Expanded Food and Nutrition Education Program (EFNEP). With agency permission, researchers set up an information table in the waiting rooms of the employment center and WIC. Signage and flyers promoted a survey on health and food behaviors, but did not specifically mention beans to reduce response bias to bean consumers only. If a man expressed interest in participating, a description of the study was read to him, and verbal consent was given before he received the survey in English or Spanish based on his choice. Participants received a $\$ 3$ cash incentive at the employment center and WIC. At the EFNEP classes, men completed surveys at the end of the third session of the six-class series. Men received grocery coupons and brand-marketed notepads, pens, tote bags, etc. Data were collected in March through September 2011. The Arizona State University Institutional Review Board deemed the study exempt [\#1009005462].

\subsection{Survey Instruments}

The survey consisted of six parts: demographics, health risk factors, food behaviors, food security, attitudes and perceptions of dry and canned beans, and bean purchasing preferences. The demographic questions and the 10-item Food Behavior Checklist from the Arizona EFNEP program enrollment form were used for all sites [22,23]. Health behavior questions (smoking, physical activity, health care) were from the Behavioral Risk Factor Surveillance System (BRFSS) [24]. The six-item USDA Core Food Security Module was used to evaluate food security status [25]. Dry and canned bean attitude and perceptions Likert statements, and bean purchasing preferences were adapted from previous research $[17,19]$.

Prior to data collection, the draft survey content was reviewed with seven Extension instructional specialists and subsequently pilot tested with two focus groups of EFNEP participants $(n=24)$. After modifications from this formative evaluation, 11 more agency researchers and field staff evaluated the survey for content validity. Another group of 23 EFNEP participants completed the survey and gave post-test feedback before formal data collection began $[26,27]$.

\subsection{Statistical Analysis}

BMIs were calculated from self-reported height and weight. These raw values were classified into categories of normal, overweight, and obese [28]. Descriptive statistics were compared by race/ethnicity categories using Chi-square and ANOVA. Likert statement responses for dry and canned bean were condensed from five to three categories of 'disagree, neutral, agree' for display in tables. Principal 
components analysis was applied to the five-category Likert-type responses for four groups of questions (the 10-item Food Behavior Checklist, health benefits of beans, attitudes toward dry beans, and attitudes toward canned beans) to identify thematic clustering. Reliability analysis to develop parsimonious models resulted in four scales with good reliability (Cronbach's alpha in parentheses): positive food behaviors (0.74), bean health knowledge (0.86), general bean attitudes (0.78) and canned bean attitudes (0.76) [29]. Data entry and analysis was performed with SPSS V.23.0 (IBM, Armonk, NY, USA), and a $p$ value $<0.05$ was considered significant.

\section{Results}

The completion rate was 91.5\% (75/82). Four men (2 Native American, 2 Asian) were excluded from further analysis due to small sample size for ethnic comparisons. Sixty percent of the respondents were employment center clients, with 22\% recruited from WIC and 13\% from EFNEP. Over half of the men (53\%) identified as white, $16 \%$ as black, and $31 \%$ as Hispanic. Hispanic men were younger $(p=0.021)$, had fewer years of education $(p=0.009)$, more children in the household $(p=0.001)$, and a greater percentage were born outside of the US $(p<0.001)$ than their peers. Eighty-seven percent of all men had one or more risk factors of physical inactivity, smoking, or obesity. There were no significant differences by race/ethnicity for food security, body mass index (BMI), exercise frequency, smoking, health care coverage, or routine checkup frequency (Table 1).

Table 1. Distribution of demographic, household and health risk characteristics of Arizona men utilizing assistance programs by race/ethnicity classifications $(\mathrm{N}=71)$.

\begin{tabular}{|c|c|c|c|c|}
\hline Characteristics & Total & $\begin{array}{l}\text { White } \\
53 \%(38)\end{array}$ & $\begin{array}{c}\text { Black } \\
16 \%(11)\end{array}$ & $\begin{array}{l}\text { Hispanic } \\
31 \%(22)\end{array}$ \\
\hline & \multicolumn{4}{|c|}{$\leftarrow$ mean \pm standard deviation $(\mathrm{SD}) \rightarrow$} \\
\hline Age in years $( \pm S D) *$ & $41 \pm 12.7$ & $45 \pm 12.5$ & $42 \pm 11.2$ & $35 \pm 12.0$ \\
\hline Monthly household income \$ & $2352 \pm 1719$ & $2757 \pm 1893$ & $2225 \pm 1620$ & $1653 \pm 1165$ \\
\hline \multirow[t]{2}{*}{ Monthly amount spent on food \$ } & $329 \pm 268$ & $318 \pm 197$ & $336 \pm 421$ & $343 \pm 285$ \\
\hline & \multicolumn{4}{|c|}{$\leftarrow \% \rightarrow$} \\
\hline \multicolumn{5}{|l|}{ Years of Education ** } \\
\hline 11th grade or less & 21.1 & $7.9 \mathrm{a}$ & $18.2_{\mathrm{ab}}$ & $45.5_{\mathrm{b}}$ \\
\hline High school or equivalent & 21.1 & $18.4_{\mathrm{a}}$ & $18.2_{\mathrm{a}}$ & $27.3_{a}$ \\
\hline Some college or Associates & 31.0 & $34.2_{\mathrm{a}}$ & $45.5 \mathrm{a}$ & $18.2_{\mathrm{a}}$ \\
\hline Bachelors or Masters & 26.8 & $39.5 \mathrm{a}$ & $18.2 \mathrm{ab}$ & $9.1_{b}$ \\
\hline \multicolumn{5}{|l|}{ Marital Status } \\
\hline Single & 39.4 & 42.1 & 54.5 & 27.3 \\
\hline Married/Cohabitating & 60.6 & 57.9 & 45.5 & 72.7 \\
\hline Has children under 20 years $* *$ & 39.4 & $26.3_{\mathrm{a}}$ & $18.2_{\mathrm{a}}$ & $72.7_{b}$ \\
\hline Born in the US *** & 74.3 & $89.5 \mathrm{a}$ & $81.8_{a}$ & $42.9_{b}$ \\
\hline \multicolumn{5}{|l|}{ Food security category } \\
\hline High food security & 72.5 & 71.1 & 72.7 & 75.0 \\
\hline Low food security & 15.9 & 18.4 & 9.1 & 15.0 \\
\hline Very low food security & 11.6 & 10.5 & 18.2 & 10.0 \\
\hline \multicolumn{5}{|l|}{ BMI Category } \\
\hline Normal 18.5-24.9 & 24.6 & 26.3 & 30.0 & 19.0 \\
\hline Overweight 25.0-29.9 & 44.9 & 44.7 & 60.0 & 38.1 \\
\hline Class I obesity $>30.0-34.9$ & 17.4 & 15.8 & 10.0 & 23.8 \\
\hline Class II+ obesity $\geq 35.0+$ & 13.0 & 13.2 & 0 & 19.0 \\
\hline \multicolumn{5}{|l|}{ Exercise for $30 \mathrm{~min}$ or more } \\
\hline Almost never & 23.9 & 23.7 & 18.2 & 27.3 \\
\hline 2-4 times per month & 28.2 & 26.3 & 18.2 & 36.4 \\
\hline 2-3 times per week & 23.9 & 26.3 & 18.2 & 22.7 \\
\hline 4 or more times per week & 23.9 & 23.7 & 45.5 & 13.6 \\
\hline
\end{tabular}


Table 1. Cont.

\begin{tabular}{|c|c|c|c|c|}
\hline Characteristics & Total & $\begin{array}{c}\text { White } \\
53 \%(38)\end{array}$ & $\begin{array}{c}\text { Black } \\
16 \%(11)\end{array}$ & $\begin{array}{c}\text { Hispanic } \\
31 \%(22)\end{array}$ \\
\hline \multicolumn{5}{|l|}{ Cigarette smoking } \\
\hline Never smoked & 40.8 & 36.8 & 45.5 & 45.5 \\
\hline Quit & 21.1 & 31.6 & 9.1 & 9.1 \\
\hline Current smoker & 38.0 & 31.3 & 45.5 & 45.5 \\
\hline Has health care coverage & 54.9 & 63.2 & 54.5 & 40.9 \\
\hline \multicolumn{5}{|l|}{ Routine checkup within } \\
\hline Past year & 56.3 & 63.2 & 54.5 & 45.5 \\
\hline Past 2 years & 22.5 & 18.4 & 36.4 & 22.7 \\
\hline $3+$ years ago & 21.1 & 18.4 & 9.1 & 31.8 \\
\hline
\end{tabular}

Table 2 shows the frequency responses to the Food Behavior Checklist statements on food resource management, food safety, and nutrition practices by race/ethnicity [17]. Seventy-three percent of the men compared prices before buying food, but black and Hispanic men did this less often $(p=0.046)$. Higher percentages of black and Hispanic men reported running out of food by the end of the month than white men $(p=0.001)$. While $70 \%$ of all men said they did not leave meat or dairy out of the refrigerator for more than 2 hours, over $60 \%$ of black and Hispanic men said they thawed foods at room temperature $(p=0.028)$. Hispanic men planned meals ahead of time 'sometimes' as opposed to 'always' in contrast to their peers $(p=0.039)$. The 'positive food behavior' scale mean was higher for white men, suggesting a trend of more frequent behaviors, than among black or Hispanic men $(p=0.056)$.

Table 2. Food behavior checklist (FBC) questionnaire responses among Arizona men utilizing assistance programs by race/ethnicity category $(\% ; \mathrm{N}=71)$.

\begin{tabular}{|c|c|c|c|c|}
\hline $\begin{array}{l}\text { For These Questions, Think about } \\
\text { How Often You Do These Behaviors. }\end{array}$ & Total & $\begin{array}{l}\text { White } \\
53 \%(38)\end{array}$ & $\begin{array}{c}\text { Black } \\
16 \%(11)\end{array}$ & $\begin{array}{l}\text { Hispanic } \\
31 \%(22)\end{array}$ \\
\hline & \multicolumn{4}{|c|}{$\leftarrow \% \rightarrow$} \\
\hline \multicolumn{5}{|l|}{$\begin{array}{l}\text { Food Resource Management } \\
\text { 1. Compare prices before you buy food? * }\end{array}$} \\
\hline Do not do & 8.6 & $0_{\mathrm{a}}$ & $18.2_{\mathrm{b}}$ & $19.0_{\mathrm{b}}$ \\
\hline Seldom & 4.3 & $2.6_{\mathrm{a}}$ & $9.1_{\mathrm{a}}$ & $4.8_{\mathrm{a}}$ \\
\hline \multicolumn{5}{|l|}{ 2. Shop with a grocery list? } \\
\hline Do not do & 14.3 & 7.9 & 27.3 & 19.0 \\
\hline Seldom & 15.7 & 15.8 & 18.2 & 14.3 \\
\hline Sometimes & 20.0 & 13.2 & 18.2 & 33.3 \\
\hline Most of the time/Always & 50.0 & 63.2 & 36.4 & 33.3 \\
\hline \multicolumn{5}{|l|}{ 3. Run out of food before end of month? ** } \\
\hline Most of the time/Always & 7.1 & $2.6_{\mathrm{a}}$ & $0_{\mathrm{a}, \mathrm{b}}$ & $19.0_{\mathrm{b}}$ \\
\hline \multicolumn{5}{|l|}{ Food Safety Behaviors } \\
\hline \multicolumn{5}{|l|}{ 4. Let meat and dairy foods sit out for $>2 \mathrm{~h}$} \\
\hline Do not do or N/A & 70.0 & 76.3 & 54.5 & 66.7 \\
\hline Seldom & 20.0 & 13.2 & 36.4 & 23.8 \\
\hline Sometimes & 8.6 & 10.5 & 0 & 9.5 \\
\hline Most of the time/Always & 1.4 & 0 & 9.1 & 0 \\
\hline \multicolumn{5}{|l|}{ 5. Thaw foods at room temperature * } \\
\hline Do not do & 24.3 & $21.1_{\mathrm{a}}$ & $36.4_{\mathrm{a}}$ & $23.8_{\mathrm{a}}$ \\
\hline Seldom & 22.9 & $34.2 \mathrm{a}$ & $0_{\mathrm{b}}$ & $14.3_{\mathrm{a}, \mathrm{b}}$ \\
\hline Sometimes & 28.6 & $26.3 \mathrm{a}$ & $9.1_{\mathrm{a}}$ & $42.9 \mathrm{a}$ \\
\hline
\end{tabular}


Table 2. Cont.

\begin{tabular}{|c|c|c|c|c|}
\hline $\begin{array}{l}\text { For These Questions, Think about } \\
\text { How Often You Do These Behaviors. }\end{array}$ & Total & $\begin{array}{l}\text { White } \\
53 \%(38)\end{array}$ & $\begin{array}{c}\text { Black } \\
16 \%(11)\end{array}$ & $\begin{array}{l}\text { Hispanic } \\
31 \%(22)\end{array}$ \\
\hline \multicolumn{5}{|l|}{ 6. Plan meals ahead of time * } \\
\hline Do not do & 10.0 & $13.2_{\mathrm{a}}$ & $9.1_{\mathrm{a}}$ & $4.8 \mathrm{a}$ \\
\hline Seldom & 14.3 & $18.4_{\mathrm{a}}$ & $18.2_{\mathrm{a}}$ & $4.8_{\mathrm{a}}$ \\
\hline Sometimes & 32.9 & $23.7_{\mathrm{a}}$ & $9.1_{\mathrm{a}}$ & $61.9_{\mathrm{b}}$ \\
\hline Most of the time/Always & 42.9 & $44.7_{\mathrm{a}}$ & $63.6_{a}$ & $28.6_{a}$ \\
\hline \multicolumn{5}{|l|}{$\begin{array}{l}\text { 7. When deciding what to feed your family, how often do you } \\
\text { think about healthy food choices? }\end{array}$} \\
\hline Do not do & 7.2 & 8.1 & 9.1 & 4.8 \\
\hline Seldom & 8.7 & 8.1 & 8.1 & 9.5 \\
\hline Sometimes & 31.9 & 27.0 & 27.3 & 42.9 \\
\hline Most of the time/Always & 52.2 & 56.7 & 54.6 & 42.8 \\
\hline \multicolumn{5}{|l|}{ 8. Prepare food without adding salt. } \\
\hline Do not do & 17.1 & 15.8 & 0 & 28.6 \\
\hline Seldom & 17.1 & 10.5 & 36.4 & 19.0 \\
\hline Sometimes & 24.3 & 23.7 & 27.3 & 23.8 \\
\hline Most of the time/Always & 41.4 & 34.2 & 27.3 & 23.8 \\
\hline \multicolumn{5}{|l|}{ 9. Use the "Nutrition Facts" or food label to make food choices? } \\
\hline Do not do & 13.0 & 10.5 & 20.0 & 14.3 \\
\hline Seldom & 31.9 & 18.4 & 30.0 & 57.1 \\
\hline Sometimes & 23.2 & 26.3 & 30.0 & 14.3 \\
\hline Most of the time/Always & 31.9 & 44.7 & 20.0 & 14.3 \\
\hline \multicolumn{5}{|l|}{ 10. Children eat within $2 \mathrm{~h}$ of waking up? $\dagger$} \\
\hline Do not do & 0 & 0 & 0 & 0 \\
\hline Seldom & 8.0 & 0 & 0 & 15.4 \\
\hline Sometimes & 4.0 & 0 & 0 & 7.7 \\
\hline Most of the time/Always & 88.0 & 100 & 100 & 76.9 \\
\hline Positive food behavior scale $( \pm S D)$ (Sum of questions $1-9)$ & $3.30 \pm 0.85$ & $3.52 \pm 0.81$ & $3.10 \pm 1.0$ & $3.0 \pm 0.76$ \\
\hline
\end{tabular}

Responses to Likert-type statements on the health benefits of beans are provided in Table 3. Close to $60 \%$ of all men agreed beans improve nutrition but about $25 \%$ of black and Hispanic men disagreed $(p=0.027)$. Almost $50 \%$ of all men stated a 'neutral' response regarding the ability of eating beans to lower cholesterol, lower cancer risk, or control blood sugar. Significantly more black and Hispanic men disagreed that eating beans could 'lower bad cholesterol' ( $p=0.016)$, control blood sugar $(p=0.049)$, or are healthy for the gastrointestinal tract $(p=0.044)$. The bean health benefits scale was significantly higher for white men indicating more knowledge than black or Hispanic men $(p=0.008)$.

Table 3. Bean health benefit knowledge among Arizona men utilizing assistance programs by race/ethnicity category (percentage; $\mathrm{N}=71$ ).

\begin{tabular}{|c|c|c|c|c|}
\hline Eating Beans Can ... & Total & $\begin{array}{c}\text { White } \\
53 \%(38)\end{array}$ & $\begin{array}{c}\text { Black } \\
16 \%(11)\end{array}$ & $\begin{array}{c}\text { Hispanic } \\
31 \%(22)\end{array}$ \\
\hline & \multicolumn{4}{|c|}{$\leftarrow \% \rightarrow$} \\
\hline \multicolumn{5}{|l|}{ 1. Improve nutrition * } \\
\hline Disagree & 11.6 & $0_{\mathrm{a}}$ & $27.3_{\mathrm{b}}$ & $23.8_{b}$ \\
\hline Neutral & 29.0 & $29.7 \mathrm{a}$ & $27.3_{a}$ & $28.6_{a}$ \\
\hline Agree & 59.4 & $70.3_{a}$ & $45.5 \mathrm{a}$ & $47.6_{a}$ \\
\hline \multicolumn{5}{|l|}{ 2. Help you feel full } \\
\hline Disagree & 8.7 & 8.1 & 9.1 & 9.5 \\
\hline Neutral & 37.7 & 37.8 & 36.4 & 38.1 \\
\hline Agree & 53.6 & 54.1 & 54.5 & 52.4 \\
\hline \multicolumn{5}{|l|}{ 3. Lower bad cholesterol * } \\
\hline Disagree & 11.6 & $0_{\mathrm{a}}$ & $18.2_{\mathrm{b}}$ & $28.6_{b}$ \\
\hline Neutral & 49.3 & $51.4_{\mathrm{a}}$ & $45.5 \mathrm{a}$ & $47.6_{a}$ \\
\hline Agree & 39.1 & $48.6_{a}$ & $36.4_{\mathrm{a}}$ & $23.8_{a}$ \\
\hline
\end{tabular}


Table 3. Cont

\begin{tabular}{|c|c|c|c|c|}
\hline Eating Beans Can . & Total & $\begin{array}{l}\text { White } \\
53 \%(38)\end{array}$ & $\begin{array}{c}\text { Black } \\
16 \%(11)\end{array}$ & $\begin{array}{l}\text { Hispanic } \\
31 \%(22)\end{array}$ \\
\hline \multicolumn{5}{|l|}{ 4. Lower cancer risk } \\
\hline Disagree & 13.0 & 2.7 & 27.3 & 23.8 \\
\hline Neutral & 56.5 & 59.5 & 54.5 & 52.4 \\
\hline Agree & 30.4 & 37.8 & 18.2 & 23.8 \\
\hline \multicolumn{5}{|l|}{ 5. Control blood sugar* } \\
\hline Disagree & 17.4 & $5.4_{\mathrm{a}}$ & $27.3_{\mathrm{b}}$ & $33.3_{\mathrm{b}}$ \\
\hline Neutral & 53.6 & $56.8_{a}$ & $45.5_{a}$ & $52.4_{a}$ \\
\hline Agree & 29.0 & $37.8_{\mathrm{a}}$ & $27.3_{\mathrm{a}}$ & $14.3_{\mathrm{a}}$ \\
\hline \multicolumn{5}{|l|}{ 6. Healthy gastrointestinal tract* } \\
\hline Disagree & 10.1 & $0 \mathrm{a}$ & $27.3_{\mathrm{b}}$ & $19.0_{\mathrm{b}}$ \\
\hline Neutral & 43.5 & $45.9 \mathrm{a}$ & $36.4_{a}$ & $42.9 \mathrm{a}$ \\
\hline Agree & 46.4 & $54.1_{\mathrm{a}}$ & $36.4_{\mathrm{a}}$ & $38.1_{\mathrm{a}}$ \\
\hline \multicolumn{5}{|l|}{ 7. Help lose weight } \\
\hline Disagree & 23.2 & 10.8 & 36.4 & 38.1 \\
\hline Neutral & 36.2 & 40.5 & 36.4 & 28.6 \\
\hline Agree & 40.6 & 48.6 & 27.3 & 33.3 \\
\hline \multicolumn{5}{|l|}{ Summary Scale } \\
\hline $\begin{array}{l}\text { Knowledge of bean health benefits ** }( \pm S D) \\
\text { (Sum of questions } 1-5)\end{array}$ & $3.25 \pm 0.84$ & $3.53 \pm 0.60$ & $2.95 \pm 1.14$ & $2.90 \pm 0.87$ \\
\hline
\end{tabular}

The Likert-type statements for attitudes and perceptions of dry and canned beans are shown in Table 4 . Over $70 \%$ of men disagreed it is difficult to make food with beans and that only 'poor people' eat beans. Few men agreed that they disliked the taste of beans, that their family will not eat beans, or that their friends do not eat beans. Almost half of the men agreed beans cause intestinal gas. Only $32 \%$ agreed that dry beans take too long to prepare. Similar positive attitudes were expressed by most men for the seven canned bean statements. There were no significant differences by race/ethnicity for the statements or the general bean or canned bean scale scores.

Table 4. Attitudes, perceptions, and consumption preferences for beans among low-income Arizona men by race/ethnicity category $(\mathrm{N}=71)$.

\begin{tabular}{|c|c|c|c|c|}
\hline $\begin{array}{l}\text { Here Are Some Reasons People Have Said Prevent Them from } \\
\text { Eating or Cooking Beans. Do You Disagree, or Agree? }\end{array}$ & Total & $\begin{array}{c}\text { White } \\
53 \%(38)\end{array}$ & $\begin{array}{c}\text { Black } \\
16 \%(11)\end{array}$ & $\begin{array}{l}\text { Hispanic } \\
31 \%(22)\end{array}$ \\
\hline & \multirow{2}{*}{\multicolumn{4}{|c|}{$\leftarrow \% \rightarrow$}} \\
\hline \multicolumn{2}{|l|}{ 1. Difficult to make food with beans } & & & \\
\hline Disagree & 76.5 & 64.9 & 100.0 & 85.7 \\
\hline Neutral & 20.6 & 29.7 & 0 & 14.3 \\
\hline Agree & 2.9 & 5.4 & 0 & 0 \\
\hline \multicolumn{5}{|l|}{ 2. Family/children will not eat } \\
\hline Disagree & 54.4 & 52.8 & 45.5 & 61.9 \\
\hline Neutral & 36.8 & 36.1 & 54.5 & 28.6 \\
\hline Agree & 8.8 & 11.1 & 0 & 9.5 \\
\hline \multicolumn{5}{|l|}{ 3. Do not like taste of beans } \\
\hline Disagree & 72.5 & 73.0 & 72.7 & 71.4 \\
\hline Neutral & 14.5 & 13.5 & 9.1 & 19.0 \\
\hline Agree & 13.0 & 13.5 & 18.2 & 9.5 \\
\hline \multicolumn{5}{|l|}{ 4. Only poor people eat beans } \\
\hline Disagree & 75.4 & 73.0 & 72.7 & 81.0 \\
\hline Neutral & 23.2 & 27.0 & 18.2 & 19.0 \\
\hline Agree & 1.4 & 0.0 & 9.1 & 0.0 \\
\hline \multicolumn{5}{|l|}{ 5. Beans not eaten by friends } \\
\hline Disagree & 68.1 & 62.2 & 81.8 & 71.4 \\
\hline Neutral & 29.0 & 32.4 & 18.2 & 28.6 \\
\hline Agree & 2.9 & 5.4 & 0 & 0 \\
\hline
\end{tabular}


Table 4. Cont.

\begin{tabular}{|c|c|c|c|c|}
\hline $\begin{array}{l}\text { Here Are Some Reasons People Have Said Prevent Them from } \\
\text { Eating or Cooking Beans. Do You Disagree, or Agree? }\end{array}$ & Total & $\begin{array}{l}\text { White } \\
53 \%(38)\end{array}$ & $\begin{array}{c}\text { Black } \\
16 \%(11)\end{array}$ & $\begin{array}{l}\text { Hispanic } \\
31 \%(22)\end{array}$ \\
\hline \multicolumn{5}{|l|}{ 6. Beans cause intestinal gas } \\
\hline Neutral & 37.7 & 43.2 & 27.3 & 33.3 \\
\hline Agree & 47.8 & 48.6 & 54.5 & 42.9 \\
\hline \multicolumn{5}{|l|}{ 7. Dry beans take too long to prepare } \\
\hline Agree & 32.4 & 32.4 & 20.0 & 38.1 \\
\hline \multicolumn{5}{|l|}{ 8. Canned beans not true to culture } \\
\hline Disagree & 71.0 & 67.6 & 72.7 & 76.2 \\
\hline Neutral & 24.6 & 29.7 & 18.2 & 19.0 \\
\hline \multicolumn{5}{|l|}{ 9. Family will not eat canned beans } \\
\hline Disagree & 61.2 & 55.6 & 81.8 & 60.0 \\
\hline Neutral & 26.9 & 27.8 & 18.2 & 30.0 \\
\hline Agree & 11.9 & 16.7 & 0 & 10.0 \\
\hline \multicolumn{5}{|l|}{ 10. If I cannot cook beans myself will go without } \\
\hline Disagree & 60.9 & 62.2 & 54.5 & 61.9 \\
\hline Neutral & 27.5 & 27.0 & 27.3 & 28.6 \\
\hline Agree & 11.6 & 10.8 & 18.2 & 9.5 \\
\hline \multicolumn{5}{|l|}{ 11. Canned beans are not healthy } \\
\hline Disagree & 57.4 & 62.2 & 50.0 & 52.4 \\
\hline Neutral & 30.9 & 27.0 & 40.0 & 33.3 \\
\hline Agree & 11.8 & 10.8 & 10.0 & 14.3 \\
\hline \multicolumn{5}{|l|}{ 12. Canned beans are too expensive } \\
\hline Disagree & 56.7 & 56.8 & 66.7 & 52.4 \\
\hline Neutral & 34.3 & 35.1 & 11.1 & 42.9 \\
\hline Agree & 9.0 & 8.1 & 22.2 & 4.8 \\
\hline \multicolumn{5}{|l|}{ 13. Canned beans do not taste good } \\
\hline Disagree & 47.1 & 55.6 & 27.3 & 42.9 \\
\hline Neutral & 29.4 & 25.0 & 45.5 & 28.6 \\
\hline Agree & 23.5 & 19.4 & 27.3 & 28.6 \\
\hline \multicolumn{5}{|l|}{ 14. Canned beans have preservatives } \\
\hline Disagree & 17.9 & 13.5 & 30.0 & 20.0 \\
\hline Neutral & 38.8 & 48.6 & 30.0 & 25.0 \\
\hline Agree & 43.3 & 37.8 & 40.0 & 55.0 \\
\hline Canned bean attitude scale $(\mu \pm$ SD) Summary of 8-13 $\S$ & $22.6 \pm 4.4$ & $23.0 \pm 5.1$ & $22.4 \pm 3.2$ & $22.0 \pm 3.4$ \\
\hline
\end{tabular}

$\S$ : Items reverse coded for summation.

Men's bean consumption, purchasing practices, and preferences are shown in Table 5. Significantly more black and Hispanic men purchased dry beans only $(p=0.050)$ compared to white men. Of the men who purchased beans, more white than black or Hispanic men indicated price was an important selection characteristic for dry beans $(p=0.036)$ and for canned $(p=0.017)$. Over $70 \%$ of Hispanic men who bought dry beans stated that they selected a dry bean based on tradition $(p=0.003)$ in comparison to their peers. Men were asked how often they consumed eight bean types.

Sixty-three percent stated they ate pintos more than once per month, followed by baked (31\%), black (27\%), dark red kidney (25\%), and chickpeas (21\%). Fewer than $15 \%$ of men reported eating navy, lentils, or black eyed peas more than once per month (data not shown). Of the $78 \%$ of men who reported buying canned beans, $36 \%$ did not purchase a specific brand, $20 \%$ did not remember the brand name, $17 \%$ bought Bush Brothers \& Company (Knoxville, TN, USA) and $14 \%$ mentioned Rosarita (Conagra, Chicago, IL, USA) (data not shown). 
Table 5. Bean consumption frequencies, purchasing practices, and preferred characteristics of low-income Arizona men by race/ethnicity $(\% ; \mathrm{N}=71)$.

\begin{tabular}{|c|c|c|c|c|}
\hline Characteristics & Total & $\begin{array}{c}\text { White } \\
53 \%(38)\end{array}$ & $\begin{array}{c}\text { Black } \\
16 \%(11)\end{array}$ & $\begin{array}{c}\text { Hispanic } \\
31 \%(22)\end{array}$ \\
\hline \multicolumn{5}{|l|}{ Bean consumption frequency } \\
\hline Once per month or less & 21.1 & 28.9 & 18.2 & 9.1 \\
\hline $2-3$ times per month & 42.3 & 42.1 & 45.5 & 40.9 \\
\hline 1-2 times per week & 29.6 & 26.3 & 36.4 & 31.8 \\
\hline $3+$ times per week & 7.0 & 2.6 & 0 & 18.1 \\
\hline \multicolumn{5}{|l|}{ Type of bean purchased } \\
\hline Both (dry and canned) & 47.8 & $44.4_{\mathrm{a}}$ & $40.0_{\mathrm{a}}$ & $57.1_{\mathrm{a}}$ \\
\hline Dry bean only & 16.4 & $5.6 \mathrm{a}$ & $40.0_{\mathrm{b}}$ & $23.8_{\mathrm{b}}$ \\
\hline Canned bean only & 11.9 & $19.4_{\mathrm{a}}$ & $0_{\mathrm{a}}$ & $4.8_{\mathrm{a}}$ \\
\hline Does not buy beans & 23.9 & $30.6_{a}$ & $20.0_{\mathrm{a}}$ & $14.3_{\mathrm{a}}$ \\
\hline Knows how to prepare dry beans & 81.4 & 73.7 & 90.9 & 90.5 \\
\hline \multicolumn{5}{|c|}{ Frequency someone cooks dry beans in household } \\
\hline Once a month or less & 40.3 & $45.9 \mathrm{a}$ & $45.5 \mathrm{a}$ & $26.3 \mathrm{a}$ \\
\hline 2-3 times per month & 31.3 & $35.1_{\mathrm{a}}$ & $36.4 \mathrm{a}$ & $21.1_{\mathrm{a}}$ \\
\hline $1-2$ times per week & 17.9 & $10.8_{\mathrm{a}}$ & $18.2_{\mathrm{a}}$ & $31.6_{a}$ \\
\hline $3+$ times per week & 10.5 & $8.1_{\mathrm{a}}$ & $0_{\mathrm{a}}$ & $17.3_{\mathrm{a}}$ \\
\hline \multicolumn{5}{|l|}{ Of the $60.6 \%(43 / 71)$ who buy dry beans } \\
\hline \multicolumn{5}{|l|}{ Preferred packaging } \\
\hline Small bags & 53.8 & 66.7 & 37.5 & 52.9 \\
\hline Medium bags & 34.9 & 33.3 & 50.0 & 29.4 \\
\hline Large bags & 9.3 & 5.6 & 0 & 17.6 \\
\hline Loose or in bulk & 30.2 & 33.3 & 25.0 & 29.4 \\
\hline \multicolumn{5}{|l|}{ Important selection traits dry } \\
\hline Price ${ }^{*}$ & 58.1 & $32.6 \mathrm{a}$ & $4.7_{\mathrm{b}}$ & $20.9 \mathrm{ab}$ \\
\hline Tradition ** & 39.5 & $16.7 \mathrm{a}$ & $25.0_{\mathrm{a}}$ & $70.6_{\mathrm{b}}$ \\
\hline Nutritional value & 30.2 & 38.9 & 25.0 & 23.5 \\
\hline Quality & 23.3 & 22.2 & 25.0 & 23.5 \\
\hline Taste of beans & 20.9 & 22.2 & 0 & 29.4 \\
\hline Brand & 25.6 & 22.2 & 50.0 & 17.6 \\
\hline Color of beans & 14.0 & 16.7 & 12.5 & 11.8 \\
\hline Cook quickly & 14. & 11.1 & 12.5 & 17.6 \\
\hline Shape & 4.7 & 5.6 & 0 & 2.3 \\
\hline \multicolumn{5}{|c|}{ Prefer to buy dry beans from a specific country? } \\
\hline No country preference & 60.5 & 50.0 & 50.0 & 76.5 \\
\hline Yes, Mexico or Central America & 16.3 & 11.1 & 12.5 & 23.5 \\
\hline Yes, USA or other & 23.3 & 38.9 & 37.5 & 0 \\
\hline \multicolumn{5}{|l|}{ Of $91.5 \%(65 / 71)$ who buy canned beans } \\
\hline \multicolumn{5}{|l|}{ Characteristics of canned beans } \\
\hline Price ${ }^{*}$ & 58.1 & $71.8 \mathrm{a}$ & $33.3_{\mathrm{ab}}$ & $35.3_{b}$ \\
\hline Taste of beans & 43.5 & 38.5 & 50.0 & 52.9 \\
\hline Quality & 28.6 & 35.9 & 14.3 & 17.6 \\
\hline Nutritional value & 17.7 & 17.9 & 33.2 & 11.8 \\
\hline Tradition-always buy type & 17.5 & 12.5 & 16.7 & 29.4 \\
\hline Brand & 17.7 & 20.5 & 33.3 & 5.9 \\
\hline
\end{tabular}

${ }^{*} p<0.05 ; * * 0.01$. Same subscript letters (e.g., a, b, etc.) indicate column proportions that are not significantly different from each other.

\section{Discussion}

The purpose of the current study was to explore the food behaviors, knowledge, and attitudes toward dry and canned beans among a sample of 71 low-income men. Comprehensive details were collected on health risk factors, food behavior practices, knowledge of the health benefits of beans, attitudes and perceptions toward dry and canned beans, and consumption patterns and purchasing preferences of beans. 
Most men had one or more health risk factors of physical inactivity, current smoking or obesity. In comparison to other men in Arizona, study participants were physically active (76.1\% vs. $74.5 \%$ ) about equal as men statewide [30]. The prevalence of overweight was about the same with participant obesity $(30.4 \%$ vs. $24.9 \%$,) and smoking $(29.7 \%$ vs. $21.0 \%)$ higher than for Arizona men of all income levels $(45 \%)$ [30,31].

The majority of men had several positive food behaviors such as comparing prices, not leaving meat or dairy unrefrigerated for long, and feeding children within 2 hours of waking. However, less than half shopped with a grocery list, planned meals ahead of time, or read the nutrition facts label on foods. Their responses on the Food Behavior Checklist are similar to Arizona women from the same time period and the pre-test results of Nebraska EFNEP adult enrollees [27,32]. Over a third of the men indicated they did not add salt when preparing foods. Notably, the Arizona men's behavior toward added salt was less frequent than reported in the two other studies [27,32]. Information about selecting "no salt added" canned beans, or dry beans which do not contain salt, may appeal to some low-income men.

Ethnic differences were apparent, with Hispanic men having less food security and less likely to compare food prices or use a grocery list than their peers. Many also indicated their children did not always eat within 2 hours of waking. Hispanic men had more children in their households, and less income on average, yet had equivalent monthly food expenditure to their peers. In a previous study, Hispanic women in metro-Phoenix were more food secure than non-Hispanic white women, but also did not shop with a grocery list nor always feed children promptly after waking in comparison [27]. Since food and storytelling are common themes in Hispanic and black culture, combining behavior change messages in stories and traditional cultural practices may be an appropriate way to convey healthful practices [33].

Shopping with a grocery list has been associated with a higher dietary quality and lower BMI among high-risk adults [34]. Since a high percentage of the low-income non-Hispanic men reported using grocery lists, and "sometimes" or "always" planning meals ahead of time, canned beans may be touted as a versatile option to add to a grocery list and to keep on hand for quick and easy meals.

Most men in this study identified beans as improving nutrition and increasing satiety, but only about a third knew of the well-documented health benefits of beans to lower cholesterol, aid in blood glucose control, and reduce cancer risk $[10,12,14]$. These health benefit knowledge gaps among low-income men may be mediated by race/ethnicity since more non-white men disagreed that these were known benefits. Similar studies have shown non-Hispanic whites to have greater nutrition knowledge than black, Hispanic and other non-white race/ethnic groups across income levels [35,36].

However, it is difficult to extricate the influence of education from race/ethnicity when known disparities are observed. Other research suggests that those with higher education display greater nutrition knowledge scores [35-37]. In response to the same bean health knowledge questions, less-acculturated Hispanic women in Arizona and in Iowa displayed lower nutrition knowledge levels than their bicultural or English dominant peers with more education [26,38]. While nutrition knowledge is a necessary component for healthy food behaviors, it does not appear to be a sufficient driver of food choice on its own [37-39]. Doma et al. found in a recent Canadian study of older adults that $98 \%$ felt eating beans could improve their health, but only $51.2 \%$ reported consuming beans regularly [40].

The low-income men in this study had positive attitudes about dry and canned beans. The majority of Arizona men reported eating beans, liked their taste, and thought it was not difficult to make meals with them. However, these men were not likely to meet the DGA recommended level of 2-3 cups per week since most men reported consuming beans only 2-3 times per month [2]. No difference was found in consumption frequencies by race or ethnicity, which was consistent with equivalent bean consumption frequencies between black and white men in another US study [41]. People had similar positive views of beans yet low rates of consumption in other studies conducted in Mexico, Australia, France, and Canada [42-45]. 
Food selection appears to be largely based on taste and cost, with taste cited as the biggest barrier to healthy eating [46,47]. Moreover, consumers have implicit views that "healthy" foods are thought to be less filling and may have an inverse relationship to those that are "enjoyable" [47,48]. Since men in this pilot study perceived beans as satiating and appetizing, and taste is a leading influence on food choice, focusing on taste rather than nutrition may be a beneficial area to pursue in marketing pulses [48,49]. Additional tailored nutrition education messages may highlight preferences of canned beans for white men and dry beans for black and Hispanic men. Monge et al. also found in Mexico that there was a preference among adults for dry, not canned, beans [42]. Likewise, based on these preliminary results, messaging may be tailored to Hispanic men by focusing on aspects of tradition, and to white men by emphasizing economic value.

There are several study limitations including the small sample size for the subgroups of black and Hispanic men. BMIs were calculated from self-reported height and weight and may be slightly lower than if measured directly by researchers [28]. The actual amount of beans consumed was not asked of participants. Results cannot be generalized to populations other than those surveyed.

Despite the cross-sectional convenience sample and small sample size, this study fills a research gap by providing descriptive details on health benefit knowledge, bean consumption patterns and preferences, and attitudes about beans among a diverse sample of low-income men. They are less likely to participate in health behavior research and are an understudied population [49,50]. The men were recruited to answer questions about health and food rather than specifically about beans in an effort to reduce response bias to only those who ate beans. Other topics to address in future research include food environment, selection practices, shopping and preparation behaviors of the household, facilitators and barriers to bean consumption, in what manner beans are consumed (in or outside the home, types of products, cuisine, entrée/side, etc.), and the male perspective on beans as a protein source compared to meat and dairy.

\section{Conclusions}

These findings suggest that low-income men practice some desirable food behaviors, have positive attitudes toward beans, and consume them, but lack knowledge of some health benefits of beans. Leveraging men's desirable food behaviors, positive views of beans, and increasing awareness of bean health benefits with clear, concise, and achievable messaging could improve health and make an increase in bean consumption more feasible [50].

Author Contributions: Funding acquisition and supervision, D.M.W.; Study Design, D.M.W.; Participant recruitment, screening, and data collection, D.M.W.; Data and statistical analysis, D.M.W., and M.M.H.; Writing Original draft preparation, M.M.H.; Writing-Review and Editing, M.M.H. and D.M.W.; Writing - Final Review and Approval, M.M.H. and D.M.W. All authors have read and agree to the published version of the manuscript.

Funding: Partial funding for original data collection was provided by a grant from the US Dry Bean Council to D.M.W. Research support for analysis and manuscript preparation for M.M.H. and D.M.W. was provided by the Iowa Agriculture and Home Economics Experiment Station, Ames, Iowa, Project No. IOW04002.

Conflicts of Interest: The authors declare no conflict of interest. The funders had no role in the design of the study; in the collection, analyses, or interpretation of data; in the writing of the manuscript, or in the decision to publish the results.

\section{References}

1. Centers for Disease Control and Prevention. Leading causes of Death (LCOD) by race/ethnicity, United States. 2017. Available online: https://www.cdc.gov/healthequity/lcod/index.htm (accessed on 5 February 2020).

2. U.S. Department of Health and Human Services and U.S. Department of Agriculture 2015-2020 Dietary Guidelines for Americans, 8th ed.; Office of Disease Prevention and Health Promotion: Washington, DC, USA, 2015. Available online: https://health.gov/dietaryguidelines/2015/guidelines/ (accessed on 18 December 2019).

3. Arganini, C.; Saba, A.; Comitato, R.; Virgili, F.; Turrini, A. Gender differences in food choice and dietary intake in modern western societies. In Public Health-Social and Behavioral Health; Intech: Rijeka, Croatia, 2012. 
4. Hiza, H.A.; Casavale, K.O.; Guenther, P.M.; Davis, C.A. Diet quality of Americans differs by age, sex, race/ethnicity, income, and education level. J. Acad. Nutr. Diet. 2013, 113, 297-306. [CrossRef] [PubMed]

5. Dowd, J.B.; Albright, J.; Raghunathan, T.E.; Schoeni, R.F.; LeClere, F.; Kaplan, G.A. Deeper and wider: Income and mortality in the USA over three decades. Int. J. Epidemiol. 2010, 40, 183-188. [CrossRef] [PubMed]

6. Aggarwal, A.; Monsivais, P.; Cook, A.J.; Drewnowski, A. Does diet cost mediate the relation between socioeconomic position and diet quality? Eur. J. Clin. Nutr. 2011, 65, 1059. [CrossRef] [PubMed]

7. Darmon, N.; Drewnowski, A. Does social class predict diet quality? Am. J. Clin. Nutr. 2008, 87, 1107-1117. [CrossRef] [PubMed]

8. Hall, C.; Hillen, C.; Garden Robinson, J. Composition, nutritional value, and health benefits of pulses. Cereal Chem. 2017, 94, 11-31. [CrossRef]

9. Drewnowski, A. The Nutrient Rich Foods Index helps to identify healthy, affordable foods. Am. J. Clin. Nutr. 2010, 91, 1095S-1101S. [CrossRef] [PubMed]

10. Padhi, E.M.; Ramdath, D.D. A review of the relationship between pulse consumption and reduction of cardiovascular disease risk factors. J. Funct. Foods 2017, 38, 635-643. [CrossRef]

11. Zhang, Z.; Lanza, E.; Kris-Etherton, P.M.; Colburn, N.H.; Bagshaw, D.; Rovine, M.J.; Hartman, T.J. A high legume low glycemic index diet improves serum lipid profiles in men. Lipids 2010, 45, 765-775. [CrossRef]

12. Hutchins, A.M.; Winham, D.M.; Thompson, S.V. Phaseolus beans: Impact on glycaemic response and chronic disease risk in human subjects. Br. J. Nutr. 2012, 108, S52-S65. [CrossRef]

13. Mattei, J.; Malik, V.; Wedick, N.M.; Hu, F.B.; Spiegelman, D.; Willett, W.C.; Campos, H. Reducing the global burden of type 2 diabetes by improving the quality of staple foods: The global nutrition and epidemiologic transition initiative. Glob. Health 2015, 11, 23. [CrossRef]

14. Mitchell, D.C.; Lawrence, F.R.; Hartman, T.J.; Curran, J.M. Consumption of dry beans, peas, and lentils could improve diet quality in the US population. J. Am. Diet. Assoc. 2009, 109, 909-913. [CrossRef] [PubMed]

15. Mobley, A.; Jones, J.; Rodriguez, J.; Slavin, J.; Zelman, K. Identifying practical solutions to meet America's fiber needs: Proceedings from the Food \& Fiber Summit. Nutrients 2014, 6, 2540-2551. [CrossRef] [PubMed]

16. Havemeier, S.; Erickson, J.; Slavin, J. Dietary guidance for pulses: The challenge and opportunity to be part of both the vegetable and protein food groups. Ann. N. Y. Acad. Sci. 2017, 1392, 58-66. [CrossRef] [PubMed]

17. Zamora, M.; Bernsten, R. Assessing markets for Central American products in US ethnic communities (The Common Beans Case). Revista Latinoamericana de Desarrollo Económico 2005, 4, 111-142. [CrossRef]

18. Smith, W.E.; Day, R.S.; Brown, L.B. Heritage retention and bean intake correlates to dietary fiber intakes in Hispanic mothers-Qué Sabrosa Vida. J. Am. Diet. Assoc. 2005, 105, 404-411. [CrossRef] [PubMed]

19. Winham, D.M.; Armstrong Florian, T.L. Hispanic women in EFNEP have low adherence with dietary guidelines regardless of acculturation level. J. Hunger. Environ. Nutr. 2010, 5, 498-509. [CrossRef]

20. Mattei, J.; Campos, H. Perceptions and behaviors of legume consumption among Puerto Rican adults. Health Behav. Policy Rev. 2014, 1, 38-49. [CrossRef]

21. Winham, D.M.; Tisue, M.E.; Palmer, S.M.; Cichy, K.A.; Shelley, M.C. Dry bean preferences and attitudes among Midwest Hispanic and Non-Hispanic white women. Nutrients 2019, 11, 178. [CrossRef]

22. Purdue University. Expanded Food and Nutrition Education Program Food Recall at Entry. 2012. Available online: https://www2.ag.purdue.edu/programs/hhs/efnep/Resource/Adult\%20Enrollment.pdf (accessed on 5 November 2018).

23. Anliker, J.; Willis, W.; Montgomery, S. The Development and Testing of the Behavior Checklist Questions for the EFNEP Evaluation/Reporting System. 2014. Available online: https:/nifa.usda.gov/sites/default/files/ resource/Development\%20and\%20Testing\%20of\%20the\%20Behavior\%20Checklist\%20Questions.pdf (accessed on 4 November 2018).

24. Behavioral Risk Factor Surveillance System. Behavioral Risk Factor Surveillance System Questionnaire. 2011. Available online: http://www.cdc.gov/brfss/questionnaires/pdf-ques/2011brfss.pdf (accessed on 24 January 2020).

25. Harrison, G.G.; Stormer, A.; Herman, D.R.; Winham, D.M. Development of a Spanish-language version of the US household food security survey module. J. Nutr. 2003, 133, 1192-1197. [CrossRef]

26. Winham, D.M.; Florian, T.L.A.; Thompson, S.V. Low-income US women under-informed of the specific health benefits of consuming beans. PLoS ONE 2016, 11, e0147592. [CrossRef]

27. Winham, D.M.; Palmer, S.M.; Florian, A.; Traci, L.; Shelley, M.C. Health behaviors among low-income Hispanic and non-Hispanic white women. Am. J. Health Behav. 2018, 42, 56-68. [CrossRef] [PubMed] 
28. Flegal, K.M.; Ogden, C.L.; Fryar, C.; Afful, J.; Klein, R.; Huang, D.T. Comparisons of self-reported and measured height and weight, BMI, and obesity prevalence from national surveys: 1999-2016. Obesity 2019, 27, 1711-1719. [CrossRef] [PubMed]

29. Cortina, J.M. What is coefficient alpha? An examination of theory and applications. J. Appl. Psychol. 1993, 78, 98. [CrossRef]

30. Centers for Disease Control and Prevention; National Center for Chronic Disease Prevention and Health Promotion. Division of Nutrition, Physical Activity, and Obesity. Data Trend and Maps. Available online: https://wwwdev.cdc.gov/nccdphp/dnpao/data-trends-maps/index.html (accessed on 31 August 2019).

31. Centers for Disease Control. State Tobacco Activities Tracking and Evaluation (STATE) System. Tobacco UseSurvey Data for Adults by Gender in Arizona. 2011. Available online: https://nccd.cdc.gov/STATESystem/ rdPage.aspx?rdReport=OSH_State.CustomReports\&rdAgReset=True\&rdShowModes=showResults\& rdShowWait=true\&rdPaging=Interactive\&islMeasure=110CSA (accessed on 31 August 2019).

32. Koszewski, W.; Sehi, N.; Behrends, D.; Tuttle, E. The impact of SNAP-ED and EFNEP on program graduates 6 months after graduation. J. Ext. 2011, 49, 5RIB6.

33. Winham, D.M.; Szkupinski Quiroga, S.; Underiner, T.L.; Etheridge Woodson, S.; Todd, M.A. Integration of theatre activities in cooking workshops improves healthy eating attitudes among ethnically diverse adolescents: A pilot study. ICAN Infant Child Adolesc. Nutr. 2014, 6, 99-108. [CrossRef]

34. Dubowitz, T.; Cohen, D.A.; Huang, C.Y.; Beckman, R.A.; Collins, R.L. Using a grocery list is associated with a healthier diet and lower BMI among very high-risk adults. J. Nutr. Educ. Behav. 2015, 47, 259-264. [CrossRef]

35. Levy, A.S.; Fein, S.B.; Stephenson, M. Nutrition knowledge levels about dietary fats and cholesterol: 1983-1988. J. Nutr. Educ. 1993, 25, 60-66. [CrossRef]

36. Wang, Y.; Chen, X. How much of racial/ethnic disparities in dietary intakes, exercise, and weight status can be explained by nutrition-and health-related psychosocial factors and socioeconomic status among US adults? J. Am. Diet. Assoc. 2011, 111, 1904-1911. [CrossRef]

37. Wardle, J.; Parmenter, K.; Waller, J. Nutrition knowledge and food intake. Appetite 2000, 34, $269-275$. [CrossRef]

38. Palmer, S.M.; Winham, D.M.; Hradek, C. Knowledge gaps of the health benefits of beans among low-income women. Am. J. Health Behav. 2018, 42, 27-38. [CrossRef]

39. Worsley, A. Nutrition knowledge and food consumption: Can nutrition knowledge change food behaviour? Asia Pac. J. Clin. Nutr. 2002, 11, S579-S585. [CrossRef] [PubMed]

40. Doma, K.M.; Farrell, E.L.; Leith-Bailey, E.R.; Soucier, V.D.; Duncan, A.M. Motivators, barriers and other factors related to bean consumption in older adults. J. Nutr. Gerontol. Geriatr. 2019, 38, 397-413. [CrossRef] [PubMed]

41. Bahr, P.R. Race and nutrition: An investigation of Black-White differences in health-related nutritional behaviours. Sociol. Health Illn. 2007, 29, 831-856. [CrossRef]

42. Monge, A.; Macias, L.; Campos, H.; Lajous, M.; Mattei, J. Perceptions and reasons for legume consumption in Mexico. Nutr. Food Sci. 2019, 49, 1232-1242. [CrossRef]

43. Figueira, N.; Curtain, F.; Beck, E.; Grafenauer, S. Consumer understanding and culinary use of legumes in Australia. Nutrients 2019, 11, 1575. [CrossRef]

44. Melendrez-Ruiz, J.; Buatois, Q.; Chambaron, S.; Monnery-Patris, S.; Arvisenet, G. French consumers know the benefits of pulses, but do not choose them: An exploratory study combining indirect and direct approaches. Appetite 2019, 141, 104311. [CrossRef]

45. Doma, K.M.; Farrell, E.L.; Leith-Bailey, E.R.; Soucier, V.D.; Duncan, A.M. Older adults' awareness and knowledge of beans in relation to their nutrient content and role in chronic disease risk. Nutrients 2019, 11, 2680. [CrossRef]

46. Glanz, K.; Basil, M.; Maibach, E.; Goldberg, J.; Snyder, D.A.N. Why Americans eat what they do: Taste, nutrition, cost, convenience, and weight control concerns as influences on food consumption. J. Am. Diet. Assoc. 1998, 98, 1118-1126. [CrossRef]

47. Suher, J.; Raghunathan, R.; Hoyer, W.D. Eating healthy or feeling empty? How the "healthy= less filling" intuition influences satiety. J. Assoc. Consum. Res. 2016, 1, 26-40. [CrossRef]

48. Crum, A.J.; Corbin, W.R.; Brownell, K.D.; Salovey, P. Mind over milkshakes: Mindsets, not just nutrients, determine ghrelin response. Health Psychol. 2011, 30, 424. [CrossRef] 
49. Taylor, P.J.; Kolt, G.S.; Vandelanotte, C.; Caperchione, C.M.; Mummery, W.K.; George, E.S.; Noakes, M.J. A review of the nature and effectiveness of nutrition interventions in adult males-a guide for intervention strategies. Int. J. Behav. Nutr. Phys. Act. 2013, 10, 13. [CrossRef] [PubMed]

50. Ryan, J.; Lopian, L.; Le, B.; Edney, S.; Van Kessel, G.; Plotnikoff, R.; Maher, C. It's not raining men: A mixed-methods study investigating methods of improving male recruitment to health behaviour research. BMC Public Health 2019, 19, 814. [CrossRef] [PubMed]

(C) 2020 by the authors. Licensee MDPI, Basel, Switzerland. This article is an open access article distributed under the terms and conditions of the Creative Commons Attribution (CC BY) license (http://creativecommons.org/licenses/by/4.0/). 